\title{
Morphologie der Hautkrankheiten im Kindesalter
}

\author{
Anne Kathrin Striegel, Cornelia Mauch, Iliana Tantcheva-Poór
}

\section{Übersicht}

Einleitung

Von der Morphe zur Diagnose:

Untersuchung des hautkranken

Kindes

Vorgehen bei Verdacht auf eine

Genodermatose

Ausgewählte Dermatosen:

von der Diagnose zur Morphe
367

367

\section{1}

\section{2}

\section{Einleitung}

Etwa ein Drittel der Patienten einer kinderärztlichen Praxis stellen sich mit Hautproblemen als Haupt- oder Nebenbefunde vor. Oft sind die Hauterscheinungen wichtige Hinweise für internistische Erkrankungen und/oder psychische Leiden. Sie können eine langfristige Wirkung auf die Wahrnehmung und Lebensqualität der Patienten sowie der Familie haben.

Für die Zuordnung der Dermatosen sind die genaue Betrachtung der Hauteffloreszenzen und ihre Verteilung unvermeidbar. Ihre präzise Beschreibung bleibt auch nach Jahren eine echte Herausforderung für die nicht dermatologischen Kollegen. Daher haben wir uns bemüht, einen nachvollziehbaren Algorithmus für die Untersuchung des hautkranken Kindes anhand der Morphologie zu erarbeiten. Zu den einzelnen Schritten haben wir Beispiele aus unserer kinderdermatologischen Sprechstunde herausgesucht, die seit Jahren an der Uniklinik Köln gemeinsam von der Haut- und Kinderklinik durchgeführt wird. Durch unsere Erfahrung mit Genodermatosenkindern ergab sich dabei der Umgang mit genetisch bedingten Hauterkrankungen als ein zusätzlicher Schwerpunkt.

\section{Von der Morphe zur Diagnose: Untersuchung des hautkranken Kindes}

„Was ist das Schwerste von allem? Mit den Augen zu sehen, was vor den Augen dir lieget.“

Johann Wolfgang von Goethe

Warum fallen uns die genaue Betrachtung der Hauteffloreszenzen und ihre Beschreibung so schwer? Es gibt ca. 2000 - 3000 definierbare Dermatosen, die z.T. mit ähnlichen Hauteffloreszenzen einhergehen können. Zudem kann dieselbe Dermatose mit unterschiedlichen Hauteffloreszenzen vorkommen; dies ist abhängig von:

- Form der Erkrankung

- Stadium der Erkrankung

- Patientenalter

- Lokalisation

- Vorbehandlung

\section{Beispiel Atopische Dermatitis}

- Im Säuglingsalter sind v. a. das Gesicht und die Kopfhaut mit oft nässenden, krustig belegten Arealen betroffen.

- Im Kleinkindalter sind die Hautveränderungen eher trocken mit Befall der Gelenkbeugen, Hals,
Nacken, Gesicht und Handrücken.

- Ab der Pubertät sind v. a. Augenlider, Hals und die großen Gelenkbeugen betroffen, oft sieht man dabei eine Verdickung der Oberhaut (Lichenifikation). 
Abb. 1 Apfelgeleeartige Farbe bei einer erwachsenen Patientin mit kutaner Sarkoidose.

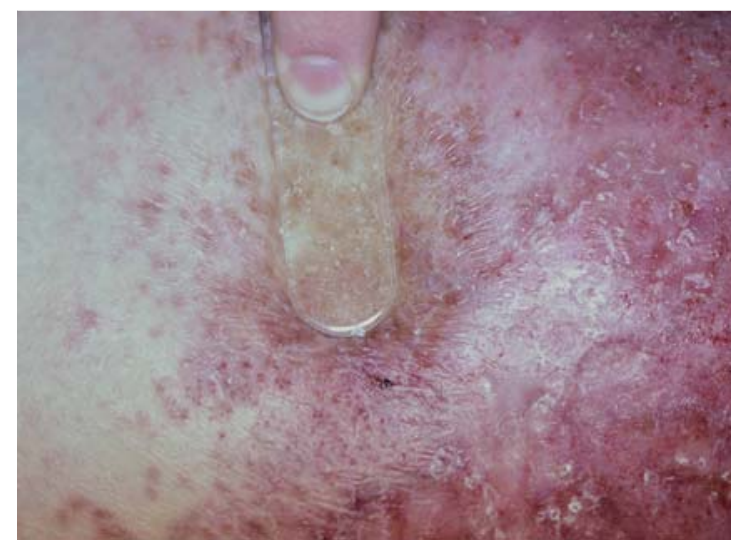

Tabelle 1

Verteilungsmuster bzw. Anordnung der Hauteffloreszenzen.

\begin{tabular}{|ll|}
\hline Verteilungsmuster/Anordnung & Beispiel \\
\hline follikulär & Folliculitis, Akne \\
\hline gruppiert & Herpesinfektion \\
\hline segmental (entlang der Dermatome) & Herpes zoster (Abb. 12) \\
\hline linear (entlang der Blashko-Linien) & $\begin{array}{l}\text { Lichen striatus (Abb. 13) } \\
\text { epidermaler Nävus (Abb. 10) }\end{array}$ \\
\hline Prädilektionsstellen & $\begin{array}{l}\text { Photodermatosen, z. B. polymorphe Licht- } \\
\text { dermatose, Phytophotodermatosen } \\
\text { Kollagenosen, z. B. LE, Dermatomyositis } \\
\text { Genodermatosen, } \text { z. B. Xeroderma pigmen- } \\
\text { tichtexponierte Areale }\end{array}$ \\
\hline tosum, Porphyrien \\
\hline Palmo-plantar & $\begin{array}{l}\text { Skabies (Abb. 5) } \\
\text { Hand-Fuß-Mund-Erkrankung } \\
\text { Erythema exsudativum multiforme (Abb. 6) }\end{array}$ \\
\hline
\end{tabular}

Beispiel: Lichtexponierte Areale

Lichtexponierte Areale sind Prädilektionsstellen für:

- Photodermatosen (polymorphe

- einige Genodermatosen (PorLichtdermatose, Phytophotodermatose) phyrie, Xeroderma pigmentosum)

- Kollagenosen (Lupus erythematodes, Dermatomyositis)
Merke: Da das Tageslicht unsere Wahrnehmung deutlich verbessert, sollte die hautärztliche Untersuchung in einem gut belichteten Raum stattfinden (am besten stellen Sie den Patienten schräg zum Fenster).

Zusätzlich braucht man ein Lineal sowie nach Möglichkeit eine Lupe und einen Glasspatel. Die Diaskopie (Untersuchung mit dem Glasspatel) erlaubt die sichere Unterscheidung zwischen einem Erythem und einer Purpura: Im Gegenteil zur Purpura ist beim Erythem das in den Gefäßen befindliche Blut wegdrückbar. Bei granulomatösen Erkrankungen ist durch den Druck mit dem Glasspatel eine apfelgeleeartige Farbe zu sehen (Abb. 1).

\section{Schritt 1: Untersuchung des gesamten Integuments}

Mit etwas Abstand sollten Sie jetzt das gesamte Integument des Patienten betrachten:

- Ist es eine Einzelläsion oder sind mehrere Läsionen vorhanden?

- Sind die Läsionen umschrieben oder generalisiert?

- Treten die Läsionen uni- oder bilateral auf?

- Sind die Läsionen symmetrisch oder asymmetrisch verteilt?

- Erkennen Sie nun bestimmte Verteilungsmuster und/ oder Prädilektionsstellen (Tab. 1)?

\section{Schritt 2: Eigenschaften der Effloreszenzen}

Konzentrieren Sie sich nun auf die einzelnen Effloreszenzen und ihre Eigenschaften. Wichtig ist hier, die Primäreffloreszenzen zu erkennen. Die Dermatologen benutzen dazu oft eine Lupe oder ein Dermatoskop. Primäreffloreszenzen entstehen de novo auf gesunder Haut. Die Sekundäreffloreszenzen gehen aus den Primäreffloreszenzen hervor (Tab. 2).

Die ABCDE-Regel, die wir bei der Unterscheidung zwischen Nävus und Melanom kennen, hilft bei der Beschreibung der Eigenschaften einzelner Effloreszenzen:

- Asymmetrie

- Begrenzung

- Colour

- Durchmesser

- Erhabenheit
Des Weiteren kommt für die Diagnostik einer Hauterkrankung erschwerend hinzu, dass jeder eine unterschiedliche Wahrnehmung für die Morphe, insbesondere ihrer Farbtöne und Begrenzung, hat. 


\section{Tabelle 2}

Effloreszenzen der Haut.

\begin{tabular}{|l|l|l|l|l}
\hline Primäreffloreszenzen & umschriebene Farbveränderung im Hautniveau \\
\hline Makula & kleine bis $0,5 / 1 \mathrm{~cm}$ große Substanzvermehrung (Abb. 3) \\
\hline Papel & $>1 \mathrm{~cm}$ flächenhafte Substanzvermehrung (Abb. 15) \\
\hline Plaque & umschriebene $>1 \mathrm{~cm}$, meist kutane/subkutane Substanzvermehrung (Abb. 9) \\
\hline Knoten & mit klarer Flüssigkeit gefüllter oberflächlicher Hohlraum (Abb. 12) \\
\hline Quaddel & mit Eiter gefüllter oberflächlicher Hohlraum (Abb. 12) \\
\hline Bläschen/Blase
\end{tabular}

\section{Sekundäreffloreszenzen}

\begin{tabular}{|c|c|}
\hline Squama/Schuppe & $\begin{array}{l}\text { Hornauflagerung in Ablösung, z. B. kleinlamellös bei Ekzemen (Abb. 7), weißlich groblamellös bei Psoriasis (Abb. 4), } \\
\text { fischschuppenartig bei Ichthyosis }\end{array}$ \\
\hline Kruste & eingetrocknetes Sekret/Blut/Eiter \\
\hline Erosio & oberflächlicher auf die Epidermis beschränkter Hautdefekt, heilt narbenlos \\
\hline Ulkus & tiefreichender Hautdefekt, narbige Abheilung \\
\hline Narbe & bindegewebiger Ersatz bei Gewebeverlust \\
\hline Atrophie & Verdünnung der Haut mit Verlust der Adnexe \\
\hline \multicolumn{2}{|c|}{ Bewährte dermatologische Begriffe } \\
\hline Ekzemmorphe & unscharf begrenztes Erythem mit kleinlamellöser Schuppung (Abb. 7) \\
\hline Exanthem & generalisierter, oft stammbetonter, zeitlich befristeter Hautausschlag mit einem dynamischen Verlauf (nach Altmeyer) \\
\hline Enanthem & Ausschlag im Bereich der Schleimhäute \\
\hline Erythrodermie & generalisierte (>90\%) Rötung der Haut \\
\hline Purpura & rötliche, nicht wegdrückbare Maculae/Papeln; bei vaskulitischer Genese sind diese meist erhaben (palpable Purpura) \\
\hline lichenoid & flechtenartig konfluierende, meist flache livide Papeln (z. B. Lichen ruber; Abb. 14) \\
\hline granulomatös & kutane Knötchen, die durch histozytäre Entzündungen ausgelöst sind, diaskopisch apfelgeleeartige Farbe (Abb.1) \\
\hline
\end{tabular}




\section{Tabelle 3}

Ausgewählte Farben als Hinweis für die Diagnose (Cave: Die Farbtöne können bei derselben Dermatose abhängig vom Stadium der Erkrankung und dem Hauttyp des Patienten variieren, z. B. erscheinen Erytheme bei dunkleren Patienten eher livid; in der Abheilung verblassen die Erytheme zunehmend).

\begin{tabular}{|l|l|}
\hline Farbe & Beispiel \\
\hline braun & $\begin{array}{l}\text { Nävuszellnävus } \\
\text { Café-au-lait-Flecken }\end{array}$ \\
\hline blau/grau & Mongolenfleck \\
\hline livid/lila & $\begin{array}{l}\text { Lichen planus (Abb. 14) } \\
\text { Kollagenosen (LE/Dermatomyositis) }\end{array}$ \\
\hline gelb & $\begin{array}{l}\text { juveniles Xanthogranulom (Abb. 15) } \\
\text { Naevus sebaceous }\end{array}$ \\
\hline weiß & $\begin{array}{l}\text { Morphea (Abb. 16) } \\
\text { Lichen sclerosus }\end{array}$ \\
\hline
\end{tabular}

In Tab. 3 sind Beispiele für charakteristische Farben aufgeführt.

Die Form der Hauteffloreszenzen kann besonders behilflich für die endgültige Zuordnung sein. Man unterscheidet u.a. folgende Formen:

- nummulär (münzförmig), z. B. nummuläres Ekzem (Abb. 7)

- targetoid (schießscheibenartig), z. B. Erythema exsudativum multiforme (Abb.6)

- anulär (randbetont), z. B. Tinea corporis (Abb. 8)

- geometrisch, z.B. bei Missbrauch, Selbstmutilation

\section{Schritt 3: Palpation der Haut}

Durch die Palpation kann man die Hautetagen besser erkennen, z.B.:

- Schuppung bei epidermaler Beteiligung

- Fluktuation bei Zysten

- Verschieblichkeit zur Unterlage bei Tumoren

Zu den bewährten Tests gehören der Nikolsky-Test und der Darier-Reibetest. Beim Nikolsky-Test kann durch tangentialen Druck die Epidermis abgeschoben werden und eine Blase wird hervorgerufen. Dieser Test wird bei der toxischen epidermalen Nekrolyse oder dem Pemphigus vulgaris positiv. Der Darier-Reibetest wird bei V.a. eine Mastozytose angewendet. Durch heftiges
Reiben auf der Hautveränderung wird eine Quaddel hervorgerufen (Abb.9).

Der Dermographismus ist eine weitere manuelle Untersuchungstechnik. Man unterscheidet weißlichen Dermographismus bei atopischer Dermatitis und urtikariellen Dermographismus bei der Urtikaria, insbesondere der Urticaria factitia.

\section{Schritt 4: Untersuchung von Haaren, Nägeln und Schleimhäuten}

Als Beispiel kann hier genannt werden, dass man bei Kindern mit ausgeprägter langwieriger Alopecia areata oft Nagelveränderungen (Nagelgrübchen, Nageldystrophie) beobachten kann.

\section{Schritt 5: Blickdiagnosen können irreführend sein}

Bevor Sie zur endgültigen Diagnose kommen, korrelieren Sie noch einmal den Hautbefund mit der Anamnese, dem Ganzkörperstatus und ggf. den Laborbefunden (Serologie, Mikrobiologie).

Auf ein paar wichtige Details in der Anamnese, die bei einer Hauterkrankung hinterfragt werden müssen, muss besonders geachtet werden:

- Wann und wo genau sind die Hautveränderungen zuerst aufgetreten?

- Gibt es Triggerfaktoren (Infekte, Arzneimittel, Licht)?

- Ist bereits eine Vorbehandlung durchgeführt worden?

- Familienanamnese?

- Reiseanamnese?

- Umgebungsanamnese (z.B. Tiere)?

\section{Beispiele: Anamnese}

- Bei einer Urtikaria bestehen die Quaddeln weniger als $24 \mathrm{~h}$ an einer Stelle.

- Bei der Skabies leiden oft andere Familienangehörige unter Juckreiz. Die Patienten wachen durch den starken Juckreiz nachts auf.

- Beim Gianotti-Crosti-Syndrom berichten die Patienten oft über einen kurz vorher durchgemachten Infekt der oberen Atemwege.

- Bei der Purpura Schönlein-Henoch bestehen neben den Petechien oftmals Gelenkschwellungen und Bauchschmerzen. 
Der Ganzkörperstatus kann Rückschlüsse auf die Hauteffloreszenzen geben. Die Lymphknoten können bei Infektions- und Tumorerkrankungen wichtige zusätzliche Informationen bringen.

\section{Schritt 6: Interdisziplinäres Vorgehen}

Bei unklaren und therapieresistenten Hautveränderungen ist die interdisziplinäre Zusammenarbeit entscheidend für die endgültige Diagnosestellung und Betreuung der Patienten. Die Bedeutung dieser Zusammenarbeit wird anhand des nächsten Kapitels illustriert.

\section{Vorgehen bei Verdacht auf eine Genodermatose}

„Nur das Seltene erweitert unsere Sinne.“

Stefan Zweig

Genodermatosen sind eine heterogene Gruppe von seltenen Hauterkrankungen, bei denen definitionsgemäß ein singuläres Gen betroffen ist (monogene Erbkrankheiten). Meist haben sie eine Prävalenz von $<1: 2000$ und gehören damit zu den sog. „orphan diseases“, deren Abklärung sehr umfangreich und kostenintensiv ist. In Deutschland leben schätzungsweise mind. 5000 Menschen mit einer seltenen Genodermatose, die sich in ca. 75\% schon im Kindesalter manifestiert.

Merke: Der chronische Verlauf, oft mit Multiorganbeteiligung, und die Betroffenheit mehrerer Familienmitglieder machen eine frühzeitige Diagnosestellung für die Einschätzung der Prognose, die komplexe Betreuung der Patienten und die Familienberatung sehr wichtig.

\section{An die „Kolibris“ denken}

Wichtige Hinweise für eine Genodermatose können sein:

- generalisierte, oft symmetrisch verteilte Hautbefunde bei Geburt insbesondere nach Ausschluss einer Infektion, z. B. Blasenbildung im Rahmen einer Epidermolysis bullosa

- multiple Pigmentflecken im 1.Lebensjahr, z.B. mehr als 6 Café-au-lait-Flecken bei NF-1

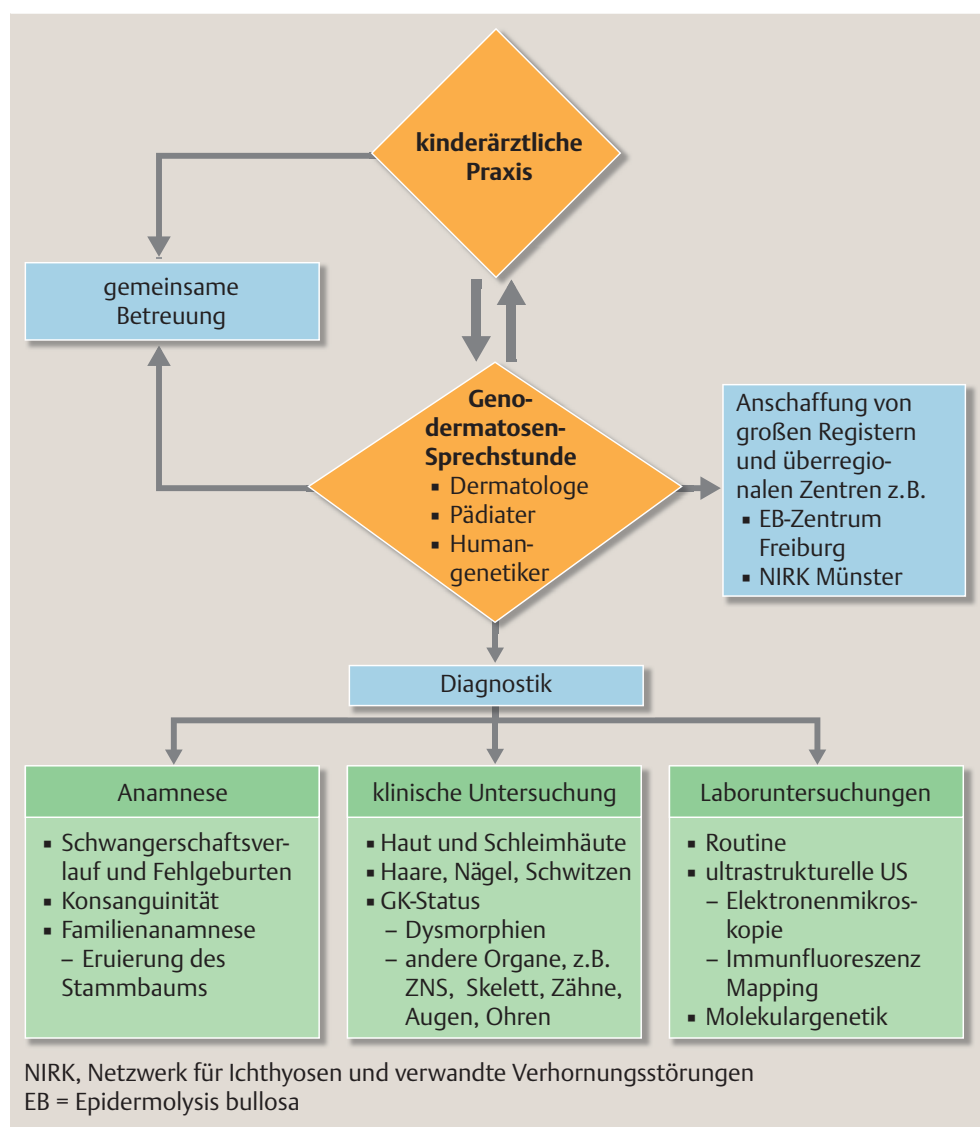

Abb. 2 Vorgehen bei Verdacht auf eine Genodermatose im Kindesalter.

- nicht zum Alter passender Hautbefund, z. B. ProgerieSyndrome

- Lichtempfindlichkeit, z.B. bei Porphyrien und Xeroderma pigmentosum

- Verteilung entlang der Blaschko-Linien als Zeichen für ein Mosaik, z. B. im Rahmen eines epidermalen Nävus (Abb. 10)

- Polymorphie der Hautbefunde, z. B. ekzematoide Dermatitis mit Petechien und Ekchymosen im Rahmen eines Wiskott-Aldrich-Syndroms

- Multisystembeteiligung, insbesondere von ZNS, Skelett, Zähnen, Augen und Ohren

- positive Familienanamnese

\section{Merke: Bei V.a. eine Genodermatose sollte eine}

Überweisung an ein spezialisiertes Zentrum erfolgen, in dem die Patienten interdisziplinär betreut werden.

In diesem spezialisierten Zentrum sollte dann eine detaillierte Befunderhebung anhand der Anamnese und der klinischen Untersuchung stattfinden (Abb. 2). 


\section{Ausgewählte Dermatosen: von der Diagnose zur Morphe}

In diesem Abschnitt werden einige Hauterkrankungen, die in den vorigen Kapiteln als Beispiele vorgestellt wurden, kurz erläutert. Diese Dermatosen haben wir aufgrund ihrer typischen Morphologie und ihrer Vielfältigkeit ausgewählt. Ausführliche Informationen über die Pathogenese, klinische Subtypen und Therapie finden Sie in den vorgeschlagenen Übersichtsarbeiten sowie in den folgenden Lehrbüchern, die sich im deutschsprachigen Raum mit mehreren Auflagen als eine unvermeidbare Hilfe für die Praxis etabliert haben:

- Höger PH. Kinderdermatologie. Differenzialdiagnostik und Therapie bei Kindern und Jugendlichen.

3. Aufl. Stuttgart: Schattauer; 2011 [1]

- Abeck D, Cremer H. Häufige Hautkrankheiten im Kindesalter. 4. Aufl. Heidelberg: Springer; 2014 [2]

\section{Gianotti-Crosti-Syndrom (Acrodermatitis papulosa eruptiva infantilis)}

Beim Gianotti-Crosti-Syndrom handelt es sich um ein reaktives, schubweise auftretendes, symmetrisches, papulöses Exanthem bei Kleinkindern (Abb.3). Es wurde primär im Rahmen einer Hepatitis-B-Vireninfektion beschrieben, tritt aber auch u. a. bei einer Epstein-BarrVirus- oder einer Coxsackie-Virusinfektion auf. Die Pathogenese ist ungeklärt, diskutiert wird eine IdReaktion auf Antigene. Nach einer kurzen Prodromalphase mit Pharyngitis und/oder gastrointestinaler Symptomatik sowie subfebrilen Temperaturen tritt das papulovesikuläre Exanthem v.a. im Bereich der Wangen, Streckseiten der Extremitäten und gluteal auf. Die Therapie erfolgt aufgrund der guten Prognose v.a. symptomatisch.

\section{Psoriasis}

Die Psoriasis ist eine häufige, akut oder chronisch verlaufende Hauterkrankung mit genetischer Disposition. Eine Assoziation mit Übergewicht und metabolischem Syndrom ist mittlerweile auch für das Kindesalter nachgewiesen. Charakteristisch sind streckseitig betonte, scharf begrenzte, rötliche Papeln oder Plaque mit einer silbrigen groblamellären Schuppung (Abb.4). Es gibt unterschiedliche klinische Formen, die geprägt sind durch:

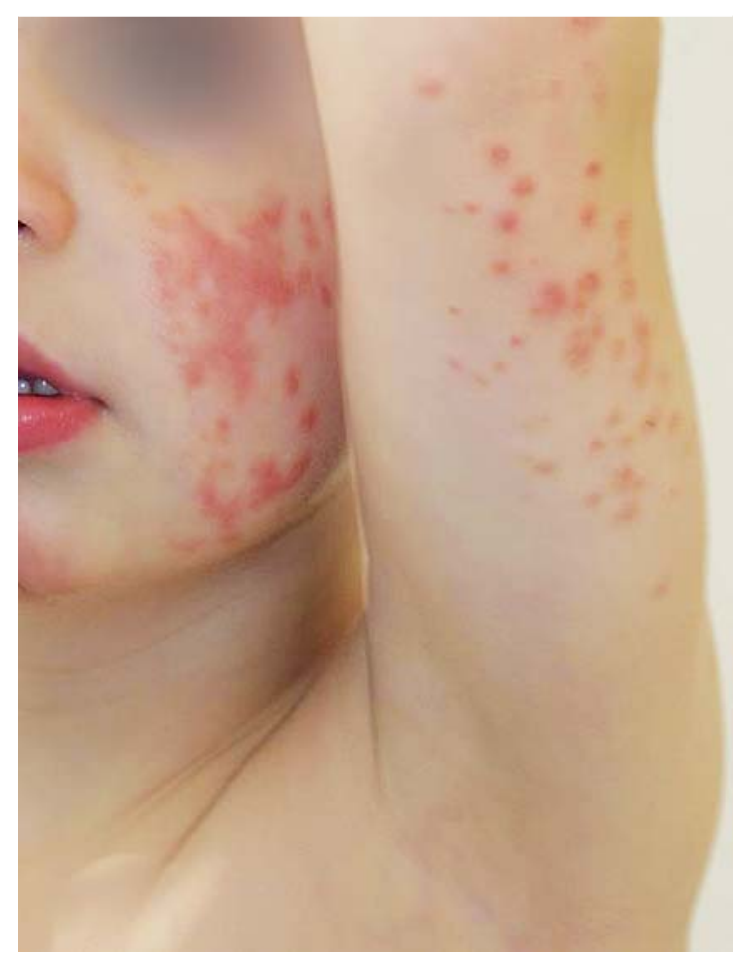

Abb. 3 Gianotti-Crosti Syndrom, auch bekannt als „infantile papulöse Akrodermatitis“. Typisch ist die Verteilung mit symmetrischem Befall der Wangen, der Streckseiten der Extremitäten und gluteal mit monomorphen erythematösen Papeln.

- Verlauf

- Lokalisation (z. B. Psoriasis inversa)

- Morphologie (Psoriasis pustulosa, Psoriasis guttata)

- weitere Organmanifestationen (z.B. Psoriasis arthropatica)

Die topische Therapie bei Kindern erfolgt nach einer ausreichenden Entschuppung mittels Dithranol, Steroiden und Vitamin-D-Präparaten. Bei nicht ausreichender Wirksamkeit bzw. einer Gelenkbeteiligung wird eine systemische Therapie primär mit Methotrexat durchgeführt, zusätzlich stehen Biologicals zur Verfügung. Differenzialdiagnotisch ist eine Abgrenzung zur atopischen Dermatitis v.a. bei jüngeren Kindern nicht immer leicht [3].

\section{Skabies}

Die Skabies ist eine häufige, weltweit verbreitete, stark juckende parasitäre Hautinfektion. Die Prävalenz der Erkrankung hängt im Wesentlichen von der Bevölkerungsdichte sowie von hygienischen Bedingungen ab. Weibliche Milben graben tunnelförmige Gänge und verbleiben 30-60 Tage lebensfähig. Klinisch symptomatisch wird eine Skabies nach 3-6 Wochen und ist 


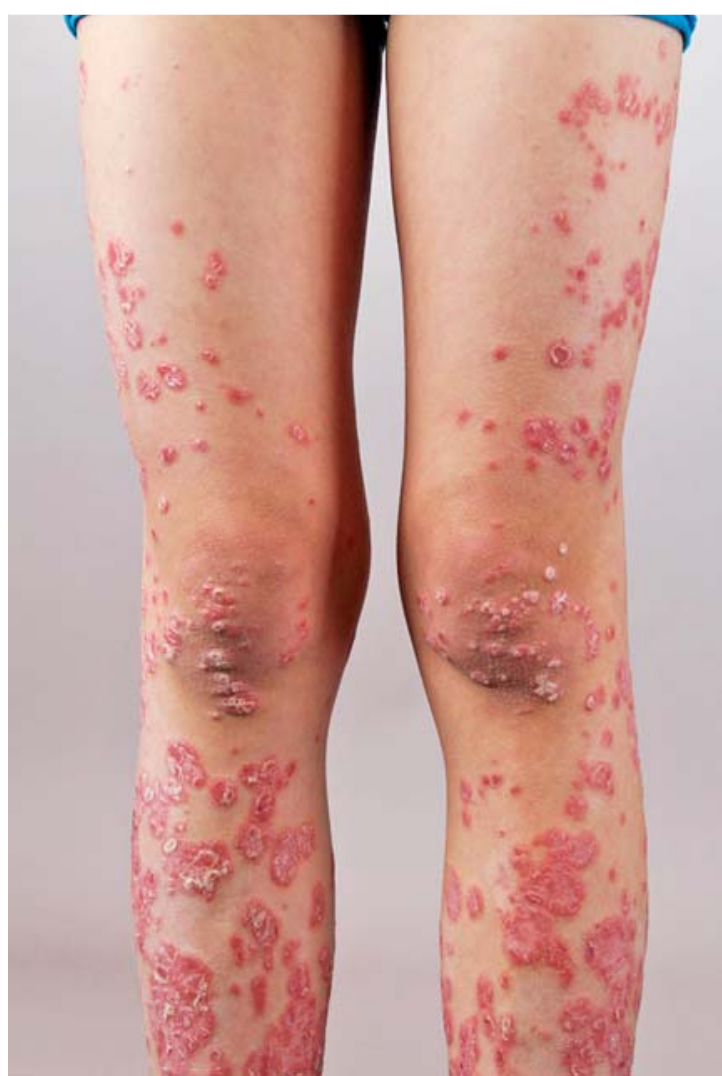

Abb.4 Psoriasis. An den Streckseiten der Extremitäten finden sich scharf begrenzte erythematöse Papeln und Plaques mit grob lamellärer silbriger Schuppung. Die Streckseiten der Extremitäten, der Haaransatz und die Ohrgänge sind typische Prädilektionsstellen für die Psoriasis. Zusätzlich können die Rima ani (Psoriasis inversa) und die Nägel (Tüpfelung, Ölflecken) befallen sein.

v.a. geprägt durch nächtlichen Juckreiz. Die papulovesikulären Hautveränderungen sind meist Ausdruck einer allergischen Reaktion des Organismus auf die Milbeninfektion (Id-Reaktion). Die Hautveränderungen sind v.a. in den Interdigitalfalten der Hände, Füße, Ellenbeugen, Kniekehlen sowie im Brustwarzenhof, Nabel und im Genitalbereich lokalisiert. Bei Säuglingen und Kleinkindern sind die Effloreszenzen oft palmar/ plantar (Abb.5) und an der Kopfhaut lokalisiert. Die Therapie erfolgt mit Permethrin 5\% in einer Cremegrundlage, ggf. muss die Therapie nach 14 Tagen wiederholt werden (der Kopf muss mitbehandelt werden!). Differenzialdiagnostisch muss man an ein Ekzem bzw. eine Impetigo denken.

\section{Erythema exsudativum multiforme}

Das Erythema exsudativum multiforme ist eine akute selbstlimitierende Dermatose mit kokardenförmigen Effloreszenzen (Abb.6). Diese tritt bei Kindern meist

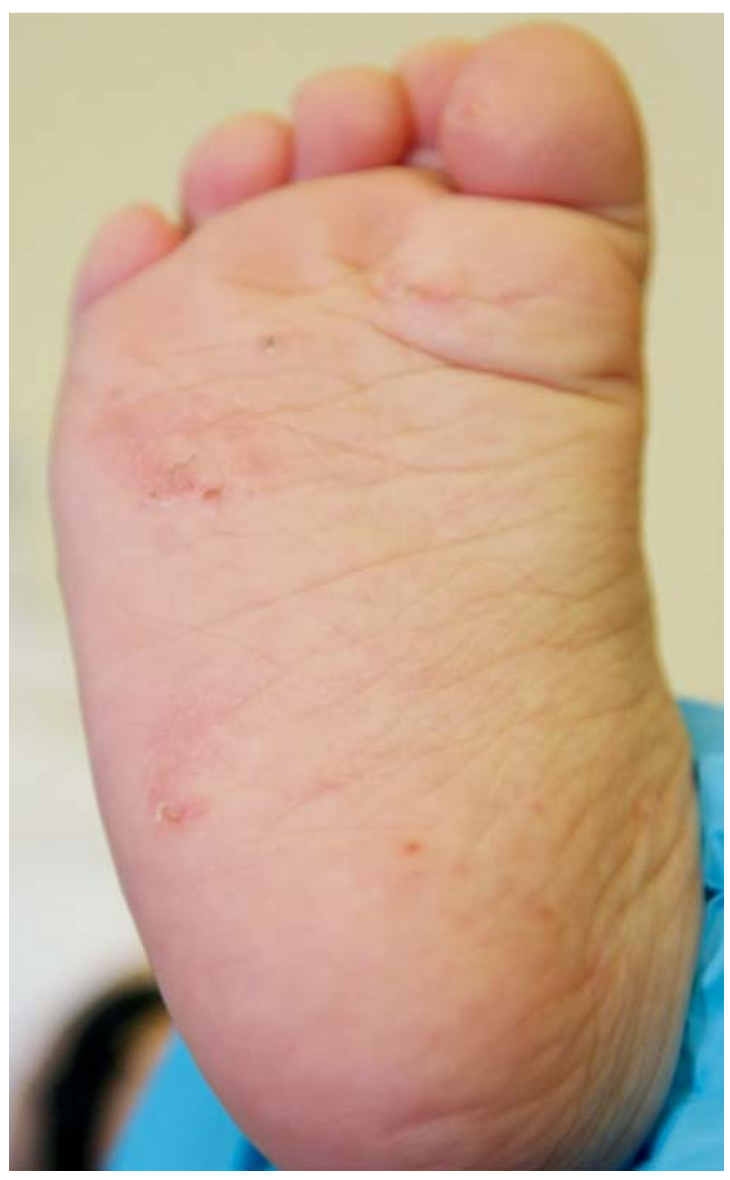

Abb. 5 Skabies. Zu sehen sind die plantar multiplen Gänge. Eine palmoplantare Beteiligung wird oft im Rahmen einer Skabies insbesondere bei Kindern, der Hand-FußMund-Erkrankung, Syphilis und dem Erythema exsudativum multiforme (Abb. 6) beobachtet.

parainfektiös auf - am häufigsten nach Herpes-simplex- und Mykoplasmeninfektionen. Die Hautveränderungen treten nach einer kurzen Prodromalphase (48-72 h) im Bereich der Hände, Fußsohlen, Nacken, Streckseiten der Extremitäten und im Gesicht auf. Die Kokarden sind rötlich-livid, teilweise hämorrhagisch und können zentral eine Blasenbildung aufweisen. Die Therapie erfolgt v.a. symptomatisch und abhängig von der Ursache antimikrobiell. Differenzialdiagnostisch muss man im Kindesalter u.a. an eine akute Urtikaria, eine Lichtdermatose oder einen Lupus erythematodes denken.

\section{Nummuläres Ekzem}

Das nummuläre Ekzem ist eine therapieresistente, stark juckende Ekzemform. Die Ursache ist polyätiologisch, im Kindesalter ist v.a. eine atopische Diathese, seltener eine mikrobielle Besiedlung (v.a. Staph. aureus) ursächlich. Die Hautveränderungen bestehen 


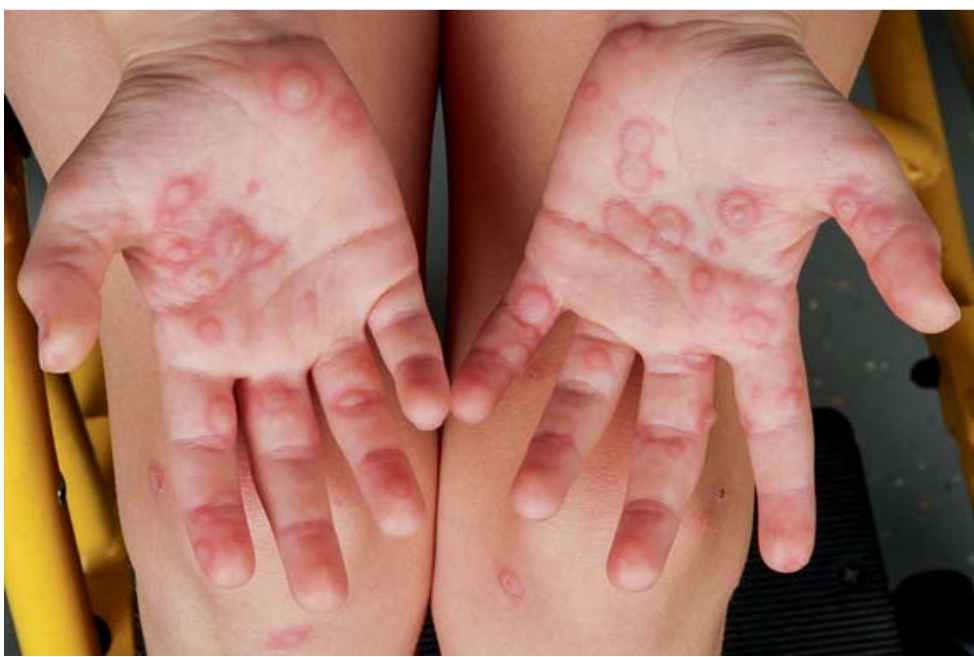

Abb. 6 Erythema exsudativum multiforme. Typische targetoide Läsionen palmar.

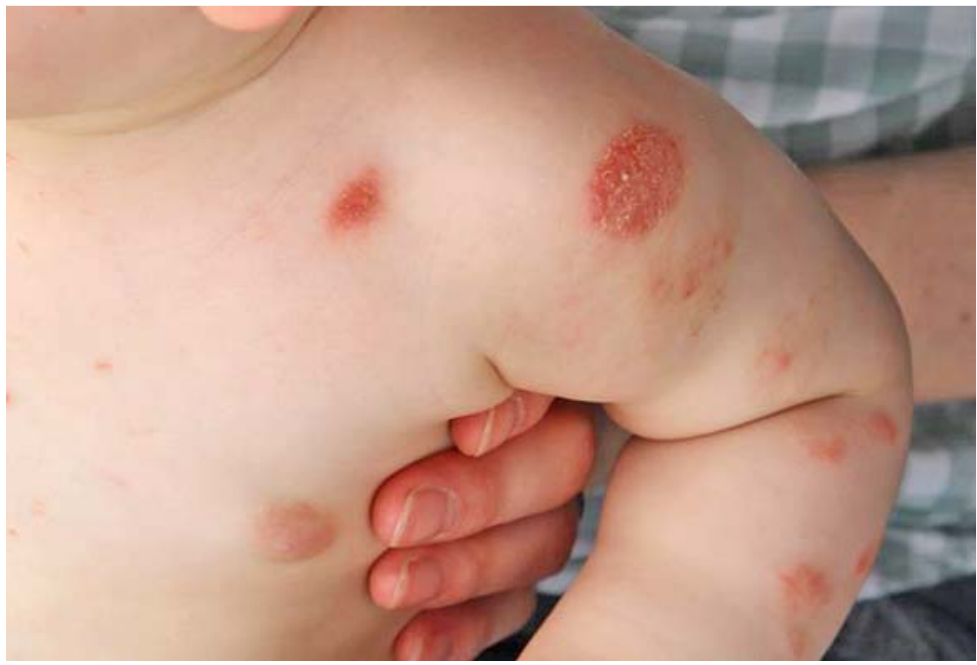

Abb.7 Nummuläres Ekzem. Disseminiert am Stamm, münzartige, unscharf begrenzte Erytheme mit kleinlamellöser Schuppung.

aus münzenartigen, teilweise „feuchten“ (erosiven), teilweise "trockenen“ (schuppigen) Plaques (Abb.7). Die Therapie erfolgt mit topischen Steroiden Klasse II, ggf. antimikrobiell und einer intensiven und regelmäßigen Hautpflege. Differenzialdiagnostisch muss man andere Ekzemtypen, eine Psoriasis und eine Tinea corporis abgrenzen.

\section{Tinea corporis}

Die Tinea corporis wird durch Dermatophyten hervorgerufen. Sie wird v.a. übertragen durch:

- Kontakt mit kontaminierten Gebrauchsgegenständen

- engen körperlicher Kontakt (Tinea gladiatorum)

- infizierte Tiere

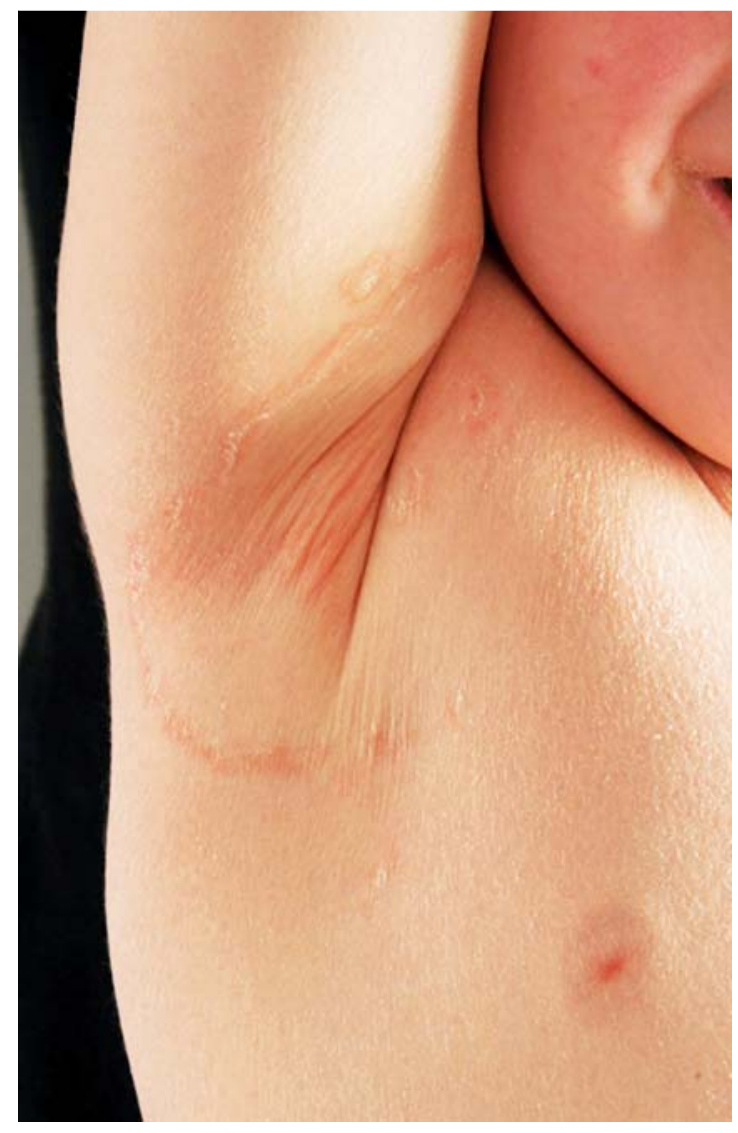

Abb.8 Tinea corporis. Axillär rechts Plaque mit anulärer/randbetonter Schuppung; zusätzlich ist eine kleinlamellöse Schuppung bei Xerosis cutis im Rahmen einer Atopie erkennbar.

Auf der Haut zeigen sich typischerweise randbetonte (anuläre) Plaques mit kleinlamellöser Schuppung (Abb.8). Für den Erregernachweis sollten Schuppen vom Randbereich nach ausreichender Desinfektion entnommen werden. Die Therapie erfolgt antimykotisch meistens topisch, bei disseminierten Befunden auch systemisch.

\section{Mastozytose}

Die Mastozytose ist eine seltene Erkrankung, die durch Mastzellanreicherung in der Haut und gelegentlich in den inneren Organen (Knochenmark, Leber, Milz) gekennzeichnet wird. In etwa 55\% der Fälle tritt diese vor dem 2. Lebensjahr auf. Bei den pädiatrischen Patienten konnten neulich als verursachend aktivierende c-kit-Mutationen in den Mastzellen nachgewiesen werden. Im Gegensatz zu Erwachsenen verläuft die Mastozytose bei Kindern selbstlimitierend (häufige spontane Rückbildung vor der Pubertät) und eine Beteiligung der inneren Organe ist extrem selten. Mastozytome (gelblich-bräunliche kutane Knoten) und die 


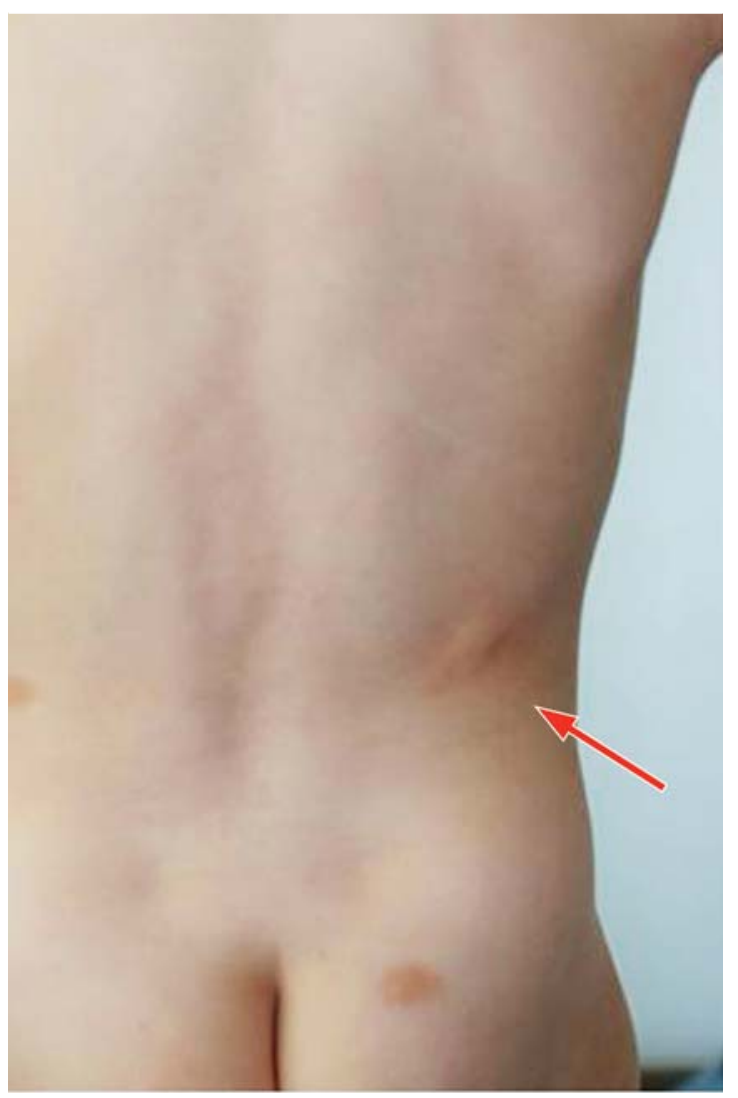

Abb.9 Mastozytose. Am Stamm gelblich-bräunliche kutane Knoten mit positivem Darier-Zeichen (Pfeil).

makulo-papulöse kutane Mastozytose (Urticaria pigmentosa) sind die häufigsten Manifestationsformen im Kindesalter; beide können gelegentlich mit einer Blasenbildung einhergehen. Typisch ist das Darier-Zeichen (Abb.9) [4].

\section{Epidermaler Nävus}

Die epidermalen Nävi sind Ausdruck eines Mosaizismus. Diese verlaufen entlang der embryonalen Wachstumslinien (Blaschko-Linien) und repräsentieren sich häufig mit streifigen bräunlichen Hyperkeratosen (Abb. 10). In seltenen Fällen können ausgeprägte Befunde syndromal auftreten und mit einer Beteiligung des Skeletts, der Augen und des ZNS einhergehen (Gruppe der sog. Epidermal-Nävus-Syndrome). Differenzialdiagnostisch sollte eine lineäre Psoriasis und Lichen striatus ausgeschlossen werden [5].

Therapeutisch kann ggf. eine ablative Lasertherapie in Erwägung gezogen werden.

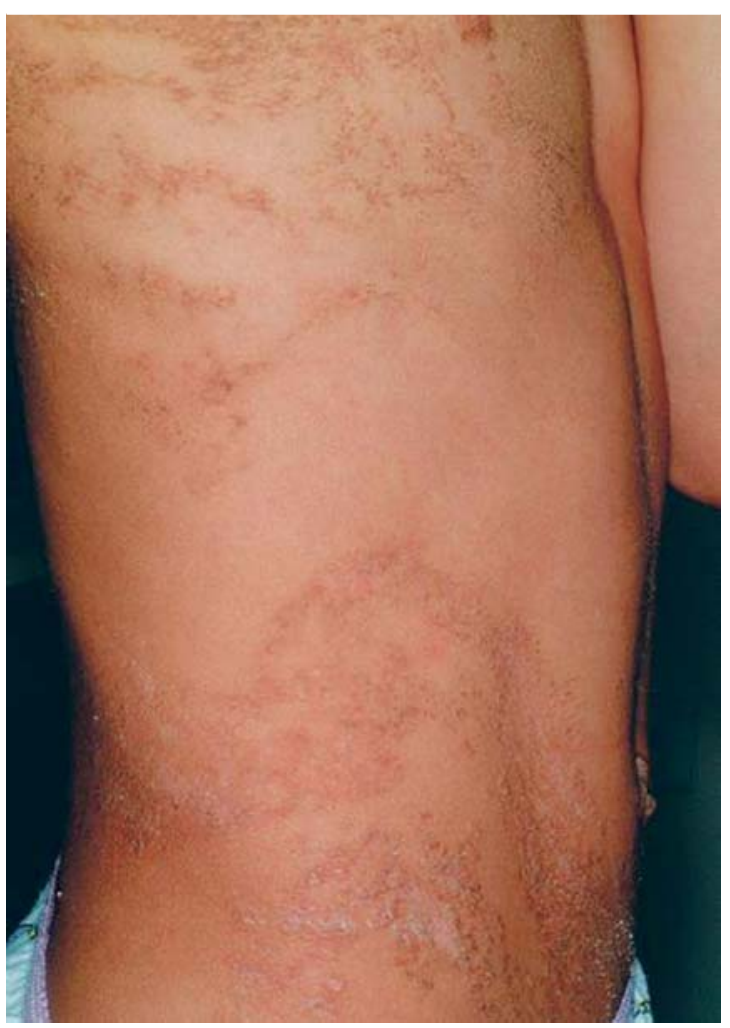

Abb. 10 Epidermaler Nävus. Am Stamm und linear entlang der Blaschko-Linien angeordnete bräunliche hyperkeratotische Papeln.

\section{Epidermolysis bullosa}

Die Epidermolysis bullosa (EB) stellt eine Gruppe von Erkrankungen dar, die durch Blasenbildung an v.a. mechanisch belasteter Haut und Schleimhaut gekennzeichnet ist (Abb. 11). Die Inzidenz wird bei ca. $1: 40000$ in Deutschland geschätzt. Ursächlich sind Genmutationen, die die Strukturproteine der epidermalen oder der dermoepidermalen Adhäsion kodieren [6].

Abhangig von der Spaltbildungsebene wird die EB in 4 Formen unterteilt, die eine unterschiedliche Ausprägung und Prognose zeigen:

- EB simplex (intraepidermale Spaltbildung)

- junktionale EB (entlang der Basalmembran)

- dystrophe EB (unterhalb der Basalmembran)

- Kindler-Syndrom (variable Spaltbildungsebene)

Merke: Insbesondere bei Neugeborenen ist eine zuverlässige Differenzierung der EB-Form aufgrund der Klinik nicht möglich. Es sollte ein Immunfluoreszenz-Mapping aus einer Hautbiopsie zwecks Bestimmung der Spaltbildungsebene über das EB-Zentrum Freiburg erfolgen. 
Abb. 11 Epidermolysis bullosa. Blasen und Milien bei einem Mädchen mit EB simplex.

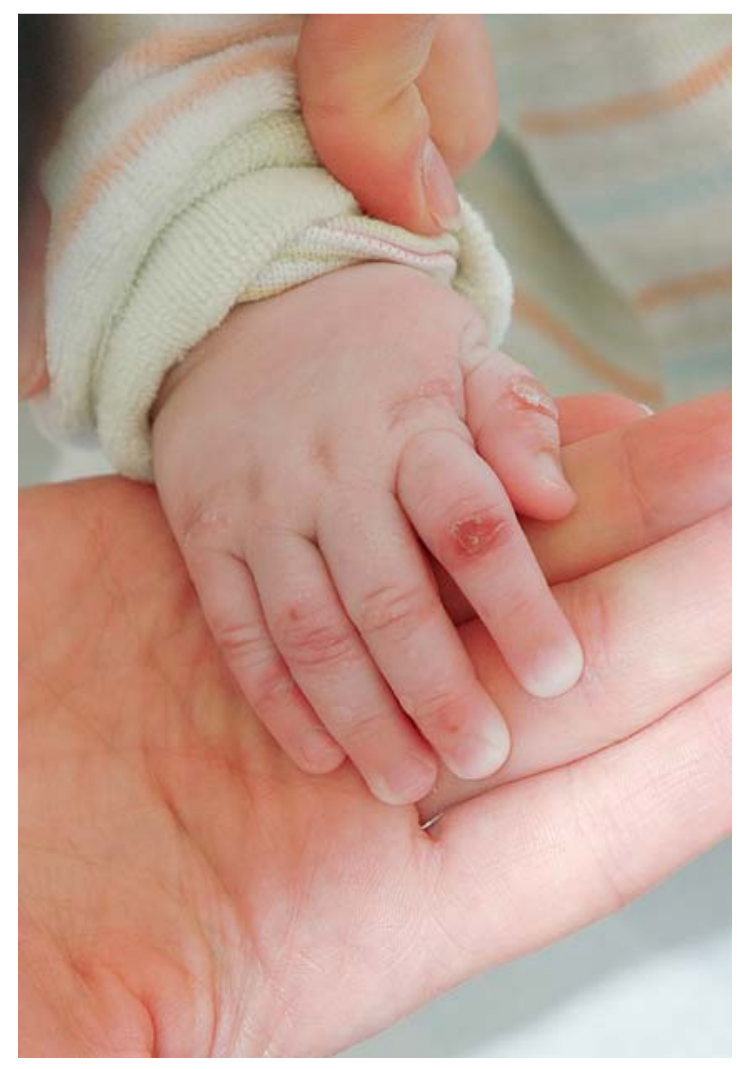

\section{Beispiel: Genodermatose}

Bei einem Säugling mit Blasenbildung und positiver Familienanamnese beim Vater und der Großmutter handelt es sich wahrscheinlich um eine autosomaldominante Epidermolysis bullosa (EB).

Die Therapie erfolgt überwiegend symptomatisch, basierend auf:

- Vermeiden von Traumata

- Vermeiden von Infektionen

- Wundmanagement

- Physiotherapie

Cave: Bei schweren Formen, die mit extrakutanen Komplikationen wie Ösophagusstrikturen, Augensynechien, Zahnschmelzdefekten und einer Malnutrition verlaufen, ist eine Anbindung an spezialisierten Sprechstunden, wo eine interdisziplinäre Mitbetreuung der Patienten gewährleistet wird, dringend zu empfehlen.

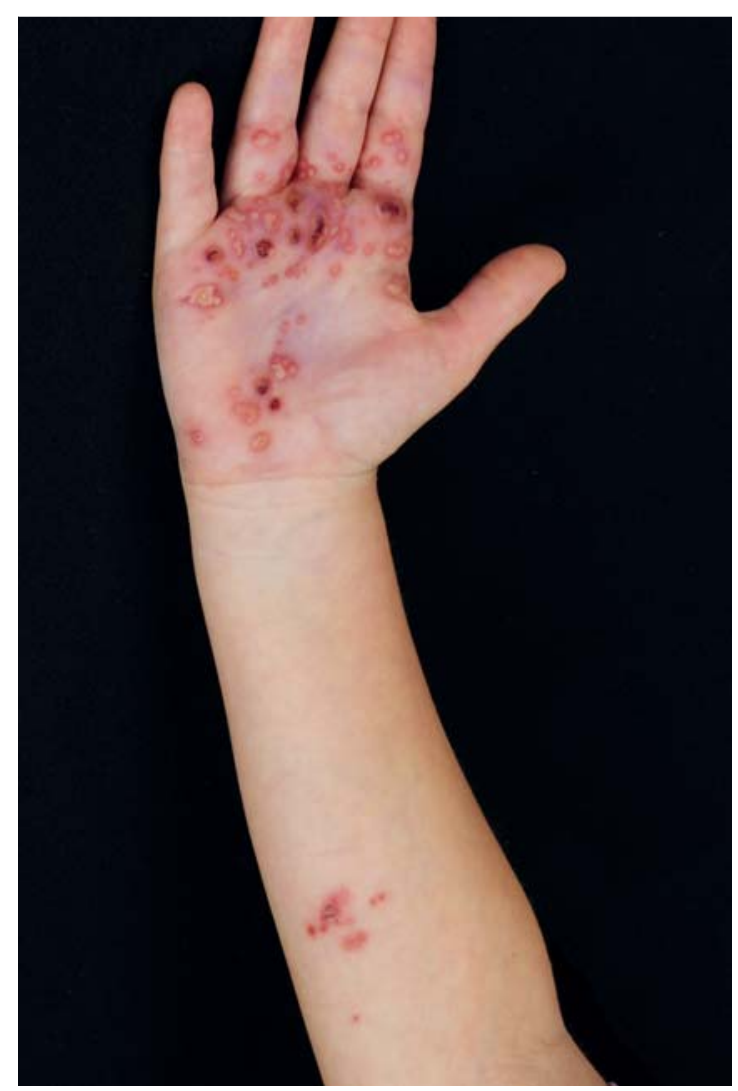

Abb. 12 Herpes zoster. Am rechten Unterarm entlang des C8Dermatoms, gruppierte Bläschen und Pusteln auf erythematösem Grund.

\section{Herpes zoster}

Der Herpes zoster ist eine neurotrope Viruskrankheit, die durch Reaktivierung des im Körper vorhandenen Varizella-zoster-Virus vorkommt; dabei kommt es zu einer Entzündung einzelner oder mehrere Spinalganglien mit gruppierten Bläschen im zugehörigen Dermatom (Abb. 12). Meist treten die Symptome einseitig auf, begleitend von brennenden Schmerzen. Parallel zu der antiviralen Therapie sollten eine adäquate Schmerztherapie und austrocknende, antiseptische Umschläge durchgeführt werden. Als Differenzialdiagnose sollten ein Erysipel und ein Herpes simplex ausgeschlossen werden.

\section{Lichen striatus}

Ein Lichen striatus ist eine den Blaschko-Linien folgende, streifenförmige Dermatose unklarer Ätiologie, die v.a. bei Kindern parainfektiös auftritt. Meistens sind die kleinen rötlichen, lichenoiden oder psoriasiformen Papeln über die gesamte Länge eines Armes bzw. eines 


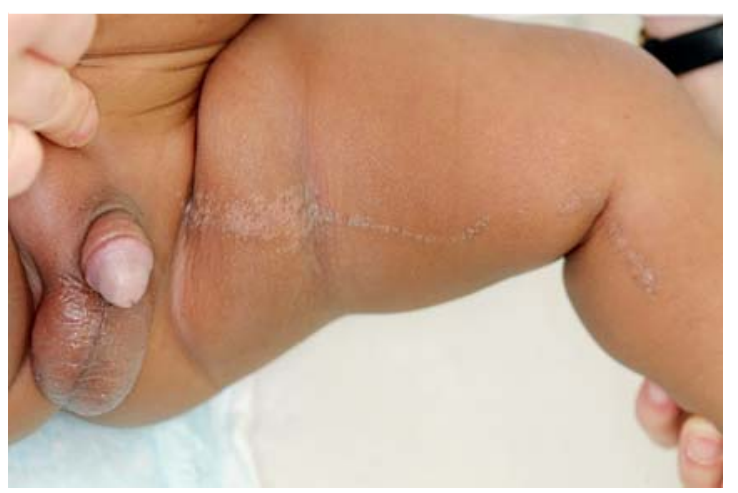

Abb. 13 Lichen striatus. Am linken Bein medial, linear angeordnete, flache, stecknadelkopfgroße, hypopigmentierte Papeln.

Beines lokalisiert (Abb. 13). Die Ausbildung der Hautveränderungen erfolgt innerhalb von $2-4$ Wochen und bildet sich nach Monaten wieder zurück. Differenzialdiagnostisch kommt u.a. ein entzündlicher linearer verruköser Nävus (ILVEN) oder ein linearer Lichen ruber in Frage.

Therapeutisch wird eine „Wait and See“-Strategie sowie eine Hautpflege empfohlen. Teilweise profitieren die Patienten auch von topischen Steroiden.

\section{Lichen ruber}

Der Lichen ruber (Knötchenflechte) ist eine nicht infektiöse, subakut bis chronisch verlaufende, juckende, selbstlimitierende (1 Monat - 10 Jahre) entzündliche Erkrankung der Haut, Schleimhäute und Adnexe (Haare/Nägel) unklarer Ätiologie (Abb.14). Es werden sowohl eine Autoimmunreakton als auch virale Antigene (Hepatitis bei Erwachsenen) ätiopathologisch diskutiert. Die Hautveränderungen erscheinen als flache, livide, stark juckende Papeln mit weißlicher Zeichnung an der Oberfläche (Wickham-Zeichnung). Häufig ist eine lineare Anordnung der Effloreszenzen in Kratz- oder Reibespuren als Köbner-Phänomen zu sehen. Die Effloreszenzen sind typischerweise an den Beugeseiten der Handgelenke lokalisiert. In 30-40\% der Fälle sind die Schleimhäute betroffen. Therapeutisch kommen mittelstarke topische Glukortikoide und Kalzineurininhibitoren zum Einsatz. Differenzialdiagnostisch sind andere lichenoide Dermatosen, z. B. ein lichenoides Arzneimittelexanthem oder lichenoide Graft-versus-Host-Reaktion, in Betracht zu ziehen.

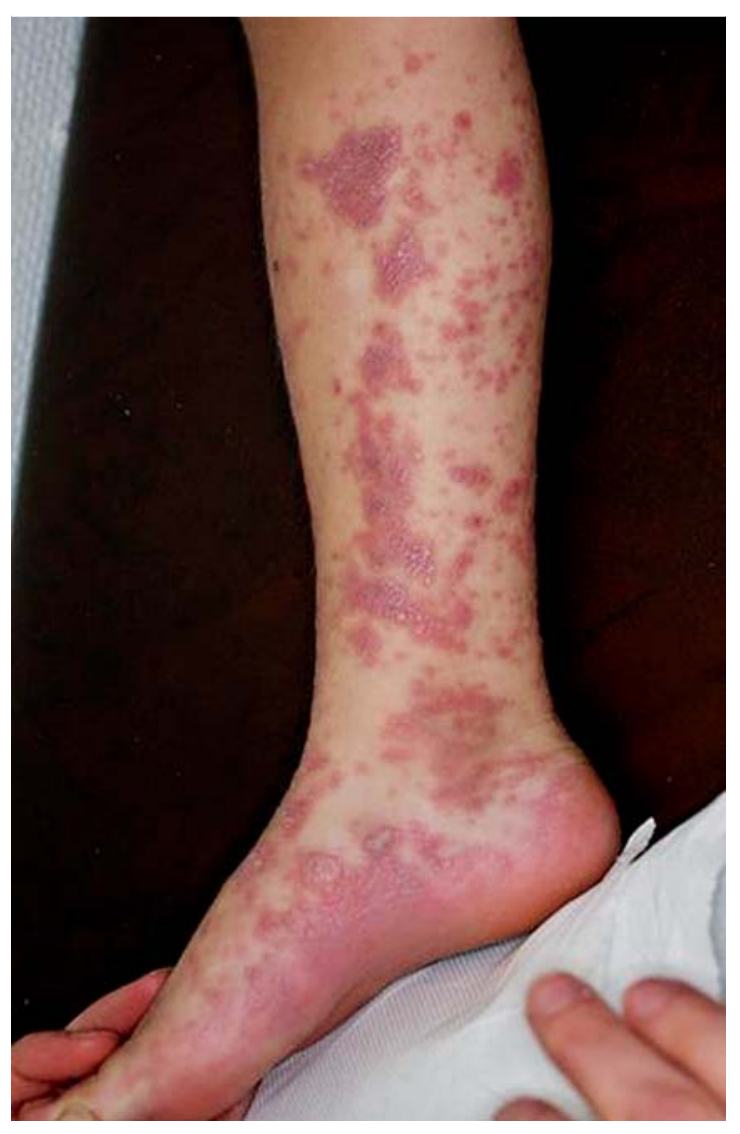

Abb. 14 Lichen

ruber. Am rechten Unterschenkel konfluierende livide flache Papeln. Diese sind z. T. durch den isomorphen Reizeffekt (Köbner-Phänomen) striär angeordnet (Cave: Das Köbner-Phänomen wird auch bei der Psoriasis beobachtet).

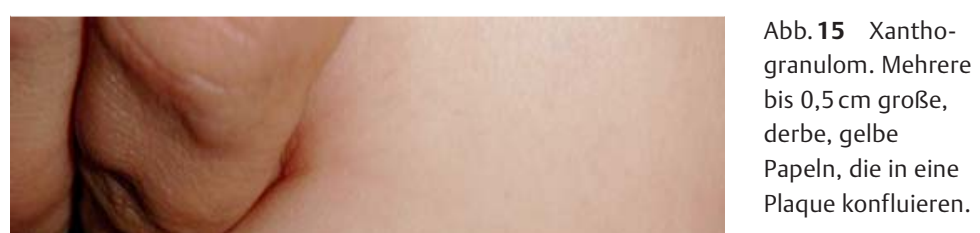


Abb. 16 Morphea. a Lineare Morphea vom En-coup-desabre-Typ in der Stirnmitte mit charakteristischer linearer Eindellung. b Morphea vom Plaque-Typ mit zentral glänzender weißlicher Sklerose und umgebendem Erythem („Iilac ring“).

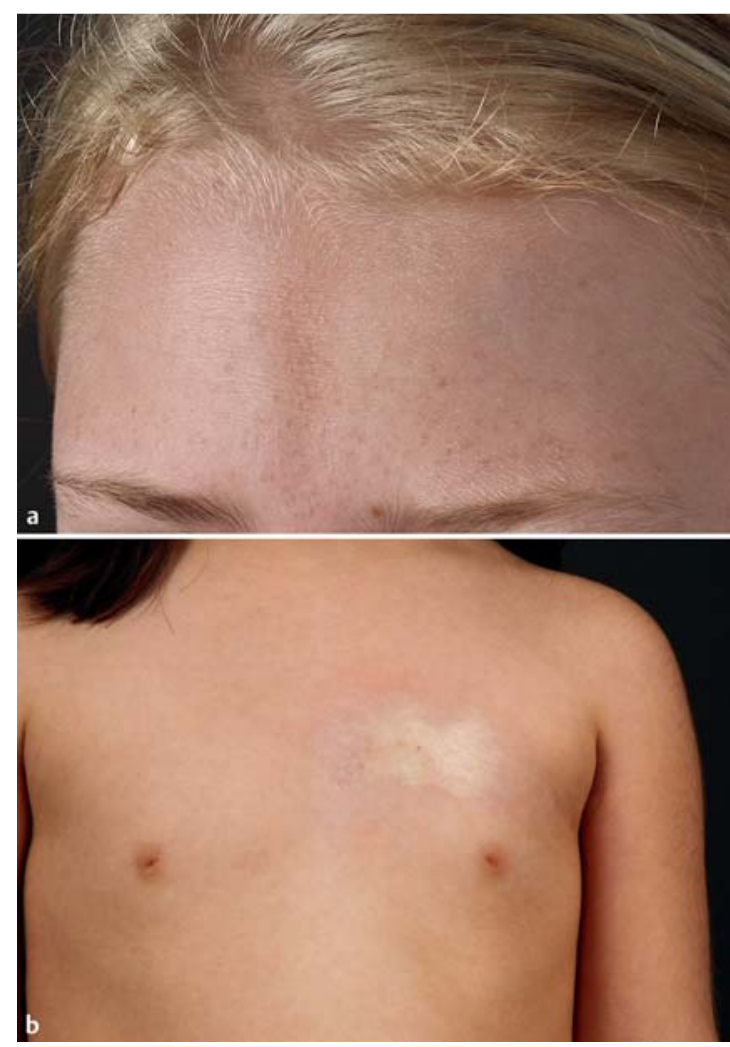

\section{Juveniles Xanthogranulom}

Das Xanthogranulom ist eine benigne selbstheilende Erkrankung im Spektrum der Nicht-LangerhanszellHistiozytosen. Überwiegend sind es solitäre, seltener multilokulär oder disseminiert auftretende, weiche, zunächst rote, dann gelbe Papeln oder Knoten, die v.a. im Bereich des Kopfes und des Stammes auftreten (Abb. 15). Meistens bilden sie sich spontan zwischen dem 1. und 6. Lebensjahr zurück. Differenzialdiagnostisch kommen u.a. Mastozytome und Nävus Spitz in Frage.

\section{Morphea/lokalisierte Sklerodermie}

Die lokalisierte Sklerodermie oder Morphea ist eine selbstlimitierende sklerosierende Bindegewebserkrankung der Haut, die in etwa $15 \%$ bei Kindern bis zum 10. Lebensjahr auftritt. Mädchen sind 2- bis 3-mal häufiger betroffen als Jungen. Je nach Ausdehnung lassen sich die folgenden 4 Formen unterscheiden:

- limitierte (zirkumskripte) Form

- lineare Form

- generalisierte Form

- tiefe Form
Im Kindesalter treten am häufigsten die lineare Form (am Kopf als „En-coup-de-sabre-Subtyp“ bekannt; Abb. 16a) und die zirkumskripte Morphea vom PlaqueTyp (Abb. 16b). Bei der linearen Form können tiefer liegende Strukturen, wie Subkutis, Muskulatur und Knochen, sowie bei Läsionen am Kopf das ZNS betroffen werden. Es sind epileptische Anfälle, Migräne, ZahnKiefer-Anomalien und okuläre Veränderungen im Rahmen des En-coup-de-sabre-Subtyps beschrieben worden. Die lineare Morphea der Extremitäten kann zu Muskel- und Knochenatrophien mit dermatogenen Kontrakturen und entzündlicher Gelenkbeteiligung führen. Bei der Morphea vom Plaque-Typ werden rundliche, meist oberflächliche Plaques mit zentraler weißlicher Verhärtung und einem umgebenden ringförmigen Erythem („lilac ring“) beobachtet. Eine interdisziplinäre Betreuung (Dermatologen, Kinderrheumatologen, ggf. Orthopäden, Zahnärzte, Krankengymnastik) ist dringend zu empfehlen, insbesondere bei den Patienten mit einer linearen Morphea [7].

\section{Fazit}

Hauterkrankungen im Kindesalter spannen im funktionellen und morphologischen Bereich einen weiten Bogen. Neben standardisiertem Vorgehen ist ein spezialisiertes Wissen gefragt, weshalb eine enge Zusammenarbeit zwischen Dermatologe und Pädiater und teilweise eine Anbindung an ein spezialisiertes Zentrum notwendig ist.

\section{Über die Autoren}

\section{Anne Kathrin Striegel}

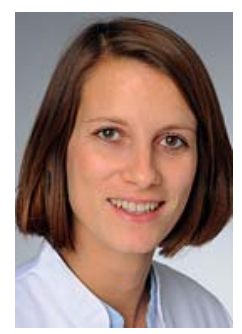

Dr. med., Jahrgang 1981. Fachärztin für Kinder- und Jugendmedizin, Kinderpneumologin. Medizinstudium in Mainz. Promotion 2007. Seit 2011 pädiatrisch-dermatologische Sprechstunde mit Frau Dr. Iliana TantchevaPoór. Assistenzärztin in der Klinik für Kinder- und Jugendmedizin der Universität Köln. Seit 2012 Fachärztin für Kinder- und Jugendmedizin, seit 2015 Kinderpneumologin. Seit 2011 Arbeit in der pädiatrisch pneumologischen und allergologischen Ambulanz der Unikinderklinik in Köln. 


\section{Cornelia Mauch}

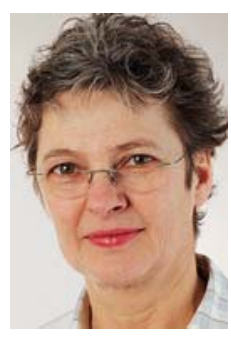

Univ.-Prof. Dr. med., Dr. rer. nat., Jahrgang 1957. Studium der Biologie und Chemie an der Universität Regensburg. 1983-1986 naturwissenschaftliche Dissertation am Max-Planck-Institut für Biochemie. 1985-1990 Studium der Humanmedizin an der Ludwig MaximiliansUniversität München. Promotion zum Dr. med. an der Medizinischen Fakultät der Ludwig Maximilians-Universität München. 1997 Habilitation und Erteilung der Venia legendi für das Fach Dermatologie und Venerologie. Seit 2001 C3-Professorin für Dermatoonkologie, leitende Oberärztin und ständige Vertreterin des Klinikdirektors.

\section{Iliana Tantcheva-Poór}

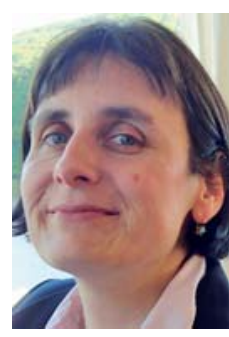

Dr. med., Jahrgang 1970. Fachärztin für Dermatologie und Venerologie. Seit 2006 pädiatrisch-dermatologische Sprechstunde sowie Genodermatosen-Sprechstunde an der Uniklinik Köln. Seit 2015 Oberärztin in der Klinik für Dermatologie und Venerologie.

\section{Literatur}

1 Höger PH. Kinderdermatologie. Differenzialdiagnostik und Therapie bei Kindern und Jugendlichen. 3. Aufl. Stuttgart: Schattauer; 2011

2 Abeck D, Cremer H. Häufige Hautkrankheiten im Kindesalter. 4. Aufl. Heidelberg: Springer; 2014

3 Höger PH, Hamm H. Psoriasis vulgaris bei Kindern und Jugendlichen. Hautarzt 2015; 66: 267 - 276

4 Siebenhaar F, Weller K, Blume-Peytavi U et al. Mastozytosen im Kindesalter. Der Hautarzt 2012; 2: 104 - 109

5 Happle R. Kutane Mosaike. Muster und molekulare Mechanismen. Dtsch Arztebl 2004; 101: A-1886/B-1575/C-1511

6 Has C, Bruckner-Tuderman L. Epidermolysis bullosa: Diagnostik und Therapie. Hautarzt 2011; 62: 82-90

7 Weibel L. Lokalisierte Sklerodermie (Morphea) im Kindesalter. Hautarzt 2012; 63: 89-96

\section{Korrespondenzadresse}

Dr. Anne Kathrin Striegel

Klinik- und Poliklinik für Kinder- und Jugendmedizin

Pädiatrische Pneumologie und Allergologie

Kerpener Str. 62

50937 Köln

E-Mail: Anne.Striegel@uk-koeln.de 


\section{CME.thieme.de}

\section{CME-Fragen}

\section{CME-Teilnahme}

- Viel Erfolg bei lhrer CME-Teilnahme unter http://cme.thieme.de

- Diese Fortbildungseinheit ist 12 Monate online für eine CME-Teilnahme verfügbar.

- Sollten Sie Fragen zur Online-Teilnahme haben, unter http://cme.thieme.de/hilfe finden Sie eine ausführliche Anleitung.

1

Was ist keine Sekundäreffloreszenz?
A Ulcus
B Macula
C Narbe
D Atrophie
E Kruste

\section{2}

Welche Aussage zu den Effloreszenzen trifft nicht zu?
A Die Papel ist eine kleine bis $0,5 / 1 \mathrm{~cm}$ große Substanzvermehrung.
B Die Quaddel ist ein flüchtiges, leicht erhabenes Erythem.
C Die Macula ist eine umschriebene Farbveränderung im Hautniveau.
D Die Schuppe ist eingetrocknetes Sekret.
E Die Pustel ist ein mit Eiter gefüllter oberflächlicher Hohlraum.

Welche Verteilung/Anordnung passt zu welcher Erkrankung?
A follikulär zu epidermalem Nävus
B linear zu Akne
C gruppiert zu Morphea
D linear zu Lichen striatus
E entlang der Blaschko-Linien zu Herpes zoster

4

Welche Farbe passt zu welcher Erkrankung?
A gelb zu Lichen sclerosus
B livid zu Nävuszellnävus
C weiß zu Morphea
D grau zu Naevus sebaceus
E braun zu Dermatomyositis

Welche Aussage zu den Prädilektionsstellen trifft nicht zu?
A Psoriasis: Streckseiten der Extremitäten, Haaransatz, Ohrgänge, Rima ani
B Erythema exsudativum multiforme: palmo-plantar, Knie- und Ellenbeugen
C Gianotti-Crosti-Syndrom: Wangen, gluteal und Streckseiten der Extremitäten
D Lichen ruber: Handgelenke, Schleimhäute
E Skabies bei Saüglingen: palmo-plantar, Kopf- und Gesichtshaut

6

Welche Aussage zu den bewährten Tests ist richtig?
A Beim Nikolski-Test entsteht durch tangentialen Druck eine Blase.
B Der Darier-Reibetest wird bei V.a. eine atopische Dermatitis angewendet.
C Weißen Dermographismus findet man bei der Urticaria factitia.
D Beim Darier-Reibetest entsteht durch heftiges Reiben eine Ulzeration.
E Unter Diaskopie versteht man die Untersuchung mit dem Dermatoskop. 


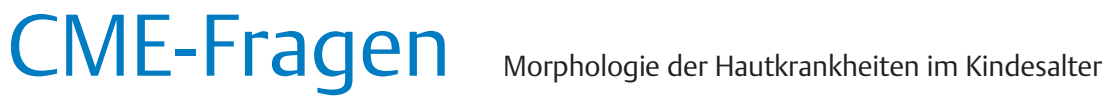

7

Welche Hautveränderung passt nicht zu der angegebenen Dermatose?
A Urtikaria: Quaddeln
B Skabies: Gänge
C Tinea corporis: nummuläre Plaques
D Erythema exsudativum multiforme: targetoide Läsionen (Kokarden)
E Herpes: gruppierte Bläschen auf erythematösem Grund

8

Welche Aussage zum GianottiCrosti-Syndrom ist falsch?
A Das Gianotti-Crosti-Syndrom wird nur bei Hepatitis-B-Viren- und Coxsakie-Virus-Infektionen beobachtet.
B Beim Gianotti-Crosti-Syndrom handelt es sich um ein parainfektiöses papulöses Exanthem bei Kleinkindern.
C Die Therapie erfolgt v.a. symptomatisch.
D Meistens gibt es eine Prodromalphase mit Pharyngitis oder Gastroenteritis.
E Das Exanthem tritt im Bereich der Wangen, Streckseiten der Extremitäten und gluteal auf.

\section{9}

Welche Aussage zum nummulären Ekzem ist richtig?
A Das nummuläre Ekzem ist nicht stark juckend.
B Ausgelöst wird diese Ekzemform v.a. durch eine virale Besiedlung der Haut.
C Therapeutisch werden v. a. Klasse-I-Steroide verwendet.
D Eine regelmäßige Hautpflege ist bei diesem Ekzemtyp nicht wichtig.
E Die Hautveränderungen bestehen aus münzartigen Plaques.

10

A Genodermatosen sind monogene Erbkrankheiten.

B Bei der Epidermolysis bullosa sind Strukturproteine der epidermalen oder epidermo-dermalen Adhäsion falsch kodiert.

C Bei Neugeborenen ist eine Subklassifizierung aufgrund der Klinik möglich.

D Das Immunfluoreszenz-Mapping ist eine schnelle Methode zur Bestimmung der Blasenbildungsebene.

E Bei Kindern mit einer Genodermatose ist die Anbindung an spezialisierte Zentren von großem Vorteil. 